\title{
INTERNATIONAL ASTRONOMICAL UNION
}

\section{COMMISSION $\theta$}

\section{INSTRUMENTS AND TECHNIQUES (INSTRUMENTS ET TECHNIQUES)}

President: J.C.Bhattacharyya

Vice-President: G.Lelievre

Organizing Committee: M. Cullum, J. Davis, C. Humphries, I.S. McLean, F. Merkle, F. Roddier, W.J. Tango, R.M. West.

\section{Introduction}

Considerable progress could be achieved in the realization of super large telescopes with apertures of 8 $\mathrm{m}$ plus during the period covered by this report. The $10 \mathrm{~m}$ Keck I telescope, the first of the twin facility utilizing active mosaic reflector has entered the operational phase, while the second unit progressed in its construction phase aiming towards its completion in 1995. Although the Columbus project of constructing a twin binocular telescope ran into some organization problems, the other projects e.g. JNLT and VLT progressed satisfactorily. Successful casting of large spun mirrors was achieved, and the possibilities of casting $8 \mathrm{~m}$ aperture light weight mirrors for future telescopes became very bright. The idea of diffraction limited image formation from large ground based telescopes became much nearer target with the employment of active mirrors in ESA's NTT. The utilization of larger fields in multiobject spectroscopy became possible with the help of multiple fibre-optic image transferring devices. Remote automatic operation of telescopes located in sites posing difficult logistics, came much nearer to realization.

Detector arrays continued becoming larger and finer with development of new chips; keeping pace with the development of computer hardware and image processing software. The spectral coverage extending deep into infra-red, created new classes of instruments in IR imaging and spectroscopy. Further developments in wide-field imaging were made by creating large area detectors by composite mosaics of buttable chips, as well as by improving scanning techniques.

In the area of high resolution imaging, several new long baseline interferometers were created, while some of the large telescope facilities nearing completion have been designed to use them as interferometers.

There have been three IAU Symposia (No. 154, 158 and 161) and one IAU Colloquium (No. 136) in which different aspects of astronomical instruments and techniques were discussed. An international conference dealing with particle streams in nature has been co-sponsored by us; it is scheduled to be held in Bangalore, India, during January 2-9, 1994. The new series of one day symposia to be held during the XXII General Assembly will also cover some of the areas of current interest in the topics covered by the Commission. Besides the above, a series of scientific meetings were held by different organizations which dealt extensively on the topics covered by our Commission. In preparation of the report, information of 21 such meetings has been taken into account. Lack of space prevents us from listing these here. 


\section{Telescopes}

\subsection{Telescopes commissioned, or under construction}

\section{Keck I telescope}

Telescopes with apertures exceeding $8 \mathrm{~m}$ became a reality when the $10 \mathrm{~m}$ Keck telescope was completed in April 1992, at Mauna Kea in Hawaii, USA. The telescope has several novel features; the primary consists 36 hexagonal segments, each $1.8 \mathrm{~m}$ across which are positioned by a computer controlled active mirror support system. The basic optical design is a f/1.75 Ritchey-Chretien, whose hyperboloidal primary is made up of a mosaic of these $1.8 \mathrm{~m}$ segments, each figured separately by following a novel technique of stressed polishing. Additional corrections to the figure are provided by mechanical stresses on individual mirrors on the telescope itself. The segments are only $75 \mathrm{~mm}$ thick, thereby achieving the ease in warping the mirror surfaces for desired figures and at the same time making it much lighter than conventional thick mirrors. Precision sensors can detect minute shifts of edges between neighbouring segments, and correct them by electromechanical actuators. The design aims at 0.25 arc second FWHM, and preliminary tests have justified the expectation. The project is a joint effort of the University of California and the California Institute of Technology; they propose to have another $10 \mathrm{~m}$ telescope in an adjoining dome, so that the twin can be used as a powerful interferometer probing ultra-high resolution structures in the celestial sources. Funding for Keck II telescope has already been assured and the telescope is expected to be completed by 1996.

The Columbus project of constructing a $8.4 \mathrm{~m}$ twin telescope to be located on Mount Graham has run into organizational problems. The project which was a joint venture between the University of Arizona, Ohio State University and Arcetri Astrophysical Observatory in Italy, was to use spin-cast light weight mirrors from the University of Arizona's mirror laboratory. But because of the Ohio State University's withdrawal from the consortium, some uncertainty of funding has crept in.

J.N.L.T. (Adapted from a report by Masanori Iye)

The Japanese project of building a $8 \mathrm{~m}$ aperture Japanese National Large Telescope has progressed well. It has been finally decided that the mirror will have an aperture of $8.2 \mathrm{~m}$. The project has been christened "Subaru"; the telescope will be located on Mauna Kea next to the Keck telescopes.

The project work has started from 1991 and expected to be completed in 9 years. Contracts were awarded in 1991 to procure the primary mirror and to design the telescope structure. The process of polishing is expected to start in 1994 (some details of the mirror in sec. 2.4).

The ground breaking at the construction site was performed in July 1992. The telescope pier and the lower part of the building are under construction at site. The manufacturing of the mechanical structure of the telescope, including center section, mirror cell and 264 actuators to support the $8 \mathrm{~m}$ mirror is in progress.

\section{V.L.T.}

The VLT project is near the half way mark, both in time and in the resources contractually committed. In this process ESO has engaged many industries in a remarkably extensive set of $R \& D$ contracts, while continuing preliminary inquiries with many for immediate future requirements. Several path breaking 
types of developments in new technology, have been achieved in international collaborations; major examples can be seen in the development of adaptive optics with several French institutions, and of remote control systems in collaboration with Trieste.

The site for the VLT has been selected at Cerro Paranal, near the NTT. It is a plateau $2632 \mathrm{~m}$ above sea level in which specific excavations for the foundations of the four $8 \mathrm{~m}$ telescopes and the control building have been dug out.

\subsection{Solar telescopes (Prepared by K.R.Sivaraman)}

The medium sized national solar telescope facility operating on Tenerife and La Palma yielding near diffraction limited images of the sun are the $50 \mathrm{~cm}$ aperture Swedish vaccum telescope, and the two German telescopes; the $45 \mathrm{~cm}$ Gregory coudé telescope and the $70 \mathrm{~cm}$ Kiepenheuer vaccum tower telescope. Another telescope projected by the French at La Palma is a dedicated telescope of $90 \mathrm{~cm}$ aperture for polarimetric measurements (THEMIS). The Earth-based Solar Telescope also planned to be located at La Palma is presently financially insecure. Similarly the Indian National Solar Vaccum Telescope project although passed through the preliminary stages of acceptance tests by the national funding agencies, has now been shelved. The Uttar Pradesh State Observatory, Nainital, India has plans for a $50 \mathrm{~cm}$ vaccum telescope.

Telescopes of similar aperture $(7 \mathrm{~cm})$ for installation at the network of six stations of the Global oscillation Network Group (GONG) together with the Michelson interferometer units are getting ready at the National Solar Observatory (NSO), Tucson. A new project titled 'Precision Solar Photometric Telescope' of the NSO, Tucson using a $15 \mathrm{~cm}$ aperture refractor and a precision photometer to study the sun's variable radiative output in the $\mathrm{Ca}$ II $\mathrm{K}$ line has received initial funding for the development of the telescope and the photometer. Images of the Fe XIV solar corona have been obtained during trials with the $15 \mathrm{~cm}$ aperture reflecting coronagraph (Mirror Advanced Coronagraph) developed at the Sacramento Peak by joint efforts from several agencies within the U.S. and the Institut d'Astrophysique, Paris. This instrument will be tested and modified further to optimise its design.

\subsection{New instrumentation facilities}

The 512 channel magnetograph which has been in operation at the Kitt Peak vaccum telescope has been replaced by the spectromagnetograph which provides better measurements. The Mc Math-Pierce telescope has now the new facility of a dual grating spectrometer with IR and visible options. At the NSO at Sacramento Peak, the Advanced Stokes Polarimeter developed by the High Altitude Observatory has been installed at the vaccum tower telescope. Another vector magnetograph using the Zeeman sensitive line ( $\mathrm{Ca}$ I $6122.2 \AA$ ) is installed at the existing $25 \mathrm{~cm}$ telescope.

A new prototype solar vector magnetograph was given preliminary trials at the Kodaikanal solar telescope. The arrangement uses a CCD at the spectrograph focal plane and measures all stokes vectors along the 6302 line; an attached P.C. computes magnetic field parameters, strengths, inclinations and azimuthal directions on a rectangular strip on the sun.

\subsection{Birefringent flters in solar observations (Contributed by A.Bhatnagar)}

With the invention of birefringent filters by Lyot in 1933 and independently by Ohman in 1935 and by 
Sloc in early thirties, the two dimensional monochromatic solar observations have been revolutionized. Since then significant contributions have been made to improve their performance by Evans at Sac Peak Observatory and building a narrow pass band filter by Ramsey, Smartt and by Giovanelli at CSIO, Australia. In recent past Alan Title at the Lockheed Observatory has made important contributions using partial polarizer to develop tunable narrow pass band $(0.1 \mathrm{~A})$ birefringent filters. During the last few years the chinese solar astronomer Ai Guoxiang has developed narrow band $(1 / 8 \AA)$ and multichannel tunable birefringent filters.

\subsection{Telescope environment and operation}

It has been observed that the "seeing" measured at a new site invariably deteriorates after the installation of the telescope. The reasons for such degradations have been traced to "dome seeing", by which the trapped air inside the telescope enclosure contributes to the deteriorating seeing quality. With a view to avoid such situations, a study group for the VLT project have designed "open" type of enclosure, in which the telescope would be largely exposed to the undisturbed windflow during observations.

In the very first conception, it was visualised that all four units of the VLT will be completely exposed during observations and protected during the day by movable roll-on/off shelters. A large wind screen, nevertheless, had the function of decreasing the wind force on the telescope. On closer examination, the idea was found not very practical and abandoned.

In the modified design, the lower part of the telescope is kept well protected in a cylindrical recess. The retractable enclosure consists of a fixed base and a rotating part. The fixed base is made of a metal space frame ring-shaped structure and supports the rotating part on a number of roller bearings. The upper rotating part is made of an approximately cylindrical panel-clad space frame, which constitutes a wind shielded recess in which the lower part of the telescope is contained. The top part, in this design, composes of a retractible hemispherical dome. This may be in the form of an inflatable dome made up of pressurized double fabric supported by rigid hoops, or a dome consisting of overlapping shell sections connected along a common axis to the rotating cylindrical part.

An alternative enclosure type which is being considered is a scaled up simplified version of the NTT building installed at La Silla. The additional advantage in this design is that this does not limit the observing elevation to $10^{\circ}-15^{\circ}$ above the horizon, but reaches much further down. It features large upside down L-shaped doors to cover the observing slit as well as louvres around the periphery of the building which allow some direct ventilation of the telescope at any azimuth.

Other studies aiming towards improvement in seeing have been reported from the $2.4 \mathrm{~m}$ Hiltner Telescope of the Michigan-Dartmouth - MTT Observatory. The primary and secondary optics have been refigured so that $\sim 80 \%$ of the light falls within a 0.4 arc second diameter circle, and to take full advantage of these the thermal properties of the telescope and dome have been remodelled. The inside dome temperature can rise $15^{\circ} \mathrm{C}$ above the night time ambient thereby heating the telescope and its optics. To offset this problem, active dome vents and an airconditioning system to cool the primary mirror have been installed. A computer collects data from thermocouples which measure the temperature of the primary mirror and other key points. The primary mirror is cooled to the predicted ambient night time air temperature using graphical data from previous nights and a computer controlled weather station. The telescope which is located on the south-west ridge of Kitt-Peak yields images of 0".7 FWHM on CCD frames exposed for $600^{3}$. 


\subsection{Telescope mirror manufacture}

For large aperture telescopes two lines of approach are being developed in two centres. ESO is experimenting on solid thin mirrors whose figure will be controlled by a closed loop servo system through electromechanical actuators. They have already employed this technique to $3.58 \mathrm{~m}$ NTT at La Silla.

The first of the four $8.2 \mathrm{~m}$ VLT mirrors has already been cast, annealed and rough ground at Schott Glaswerke, Mainz and delivered to REOSC (Recherches et études doptique et des sciences connexes) at Ballainvilliers, near Paris, France, who will produce the final mirror. Schott have set up three furnaces, the idea being that all three will be used simultaneously and the subsequent blanks will be delivered at one year intervals. REOSC have set up two machines, one for coarsely figuring the mirrors, after which the blanks will be transferred to the second machine with which the final, highly delicate polishing will be performed. After thorough testing the mirrors are scheduled to be transported to Chile at the new site at Cerro Paranal. The scientists are confident that very accurate figuring as they have achieved for the NTT mirror will also be possible.

The second line of approach is by the Steward Observatory's Mirror Laboratory (SOML) at the University of Arizona, where the mirror of borosilicate glass, a material with much lower melting point is moulded in a form with honey comb network at the back for stiffness as well as for reduced thermal inertia. Dr Angel also visualizes a system of cooling by forced draughts on the telescope. The largest blank moulded by this technique is the $6.5 \mathrm{~m}$ meant for replacing the six $1.8 \mathrm{~m}$ mirrors in the MultiMirror Telescope at Mount Hopkins. The two $8 \mathrm{~m}$ blanks for the Columbus Twin telescope project are expected to be prepared in this laboratory.

Still a third technique of mirror making has been adopted for the "Subaru" project - the Japanese Large National Telescope, scheduled to be put in operation by 1998 at the Mauna Kea Observatory. The 20 $\mathrm{cm}$ thick primary mirror will have its surface generated on a composite meniscus comprising of a top plano-plano surface backed by a bank of 44 boules of hexagonal cross section fused to this meniscus. The meniscus will be generated by sagging the top plate down in an oven; it is expected that $f / 1.8$ will be achieved in one year.

Not much progress have been reported about other techniques of producing light weight mirrors using all metal or metal foam sandwiches. The success attained by the thin glass mirrors assisted by active corrections perhaps have reduced the urgency of finding alternative approaches.

One notable success in mirror fabrication techniques has been the experiment of stressed polishing adopted in Keck telescope mirrors. All laboratory tests on the individual segments showed that the idea has been totally sound, and giant aspheric suraces can be generated by this technique. Reports on sky observations are awaited.

\section{Instruments and techniques}

The availability and promise of newer and larger telescopes have promoted activities in design and fabrication of many new instruments. A comprehensive cover of all these activities is not feasible in this small report. Attempts have been made to cover some of them, which unfold glimpses of scientific ingenuities and foresight among the instrumentation scientists. 


\subsection{Instrumentation (Prepared by S.K.Jain)}

The LOOM (Laboratoire d Optique de 1 Observatoire de Marseille) team reports the installation of a laser machine, LAMA, which will be used for producing 2D masks. This will allow greater accuracy at the slit edge and more reliability under operation. A new version of CORAVEL radial velocity meter-SUPERCORAVEL - has been designed and built jointly with Observatories de Geneve and de Haute-Provence. This instrument will operate in 1993 at OHP. The very high resolution available increased by use of optical fibers is expected to provide radial velocity accuracies upto 10 or $5 \mathrm{~m} / \mathrm{s}$. An active optics method has been developed to obtain plane-aspheric reflective gratings which now form part of a number of high-throughput spectrographs.

Pulkovo Observatory, Russia, has started work on the development of two star-measuring mechines, "FANTASIA" and "PARSEC". The former can accommodate a plate of $240 \mathrm{~mm}$ x $240 \mathrm{~mm}$, and measure 800-1500 stars/hour, with a precision of $0.00033 \mathrm{~mm}$; the latter can accommodate a plate of $160 \mathrm{~mm} \mathrm{x}$ $160 \mathrm{~mm}$, and measure 400 stars/hour with a precision of $0.001 \mathrm{~mm}$.

Two infrared array camera systems-IRCAM and MIRAC (Middle Infrared Camera) - for ground based astronomy have been reported by Shivanandan. IRCAM is based on 10 x 50 elements Si: As BIBIB/ SWIFET HYBRID array operating at 10K. MIRAC uses a $20 \times 64$ elements Si:As with a capacitance trans-impedance amplifier readout and operates at $10 \mathrm{~K}$ in a liquid helium cooled cryostat. It has an operating wavelength range of 2 to 26 microns.

Infrared Imaging Detector Lab. (UCLA, USA) has constructed a K-band survey camera for small telescopes which provides 0.5 " pixels and a 128" x 128 " field of view. This lab is also working on the development of a general-purpose, two-channel camera system designed for larger telescopes.

A high-resolution slitless spectrograph-Heterodyned Holographic spectrometer (HHS) - has been developed at Kapteyn Observatory (The Netherlands). It combines the high throughput of a Fourier Transform Spectrometer with the convenience of a CCD detector.

The instrument laboratory at Paris-Meudon Observatory, France, has developed a single object fiber spectrograph, ISIS-BIS which is the second generation version of ISIS. This spectrograph is able to achieve a resolution of 60000 . Its infrared version, ISIS-IR, has been updated with a 2-D mode and can go upto 2.2 microns. A multi-object fiber positioner has been developed for the prime focus of the 3.60 m telescope at La Silla, Chile. This has 30 independent arms which sweep a field of one degree. This laboratory has also developed a second generation cassegrain double spectrograph for the CFHT. This instrument employing MOS/SIS, has a field of 10' x 10'.

In an interesting paper (1), a team at Space Science Department, ESTEC, proposes a novel approach to the physics of optical photon detection. They demonstrate the feasibility of optical photon counting using a superconducting substrate in combination with an array of widely spaced superconducting tunnel junctions of lower energy gap. Apart from high quantum efficiency over a very broad wavelength range, without readout noise, with high time resolution, the new proposed detector will have intrinsic higher wavelength resolution, claim the researchers.

VULCAN: A low resolution spectrophotometer for measuring the integrated colors of galaxies has been developed jointly by Astronomisches Institut, Austria and Department of Astronomy, University 
of Michigan (USA). The main purpose of this instrument is to obtain narrow-band optical colors for low surface brightness galaxies, which can then be applied to the study of stellar populations in these galaxies.

Indian Institute of Astrophysics (IIA), Bangalore, has developed a Rotational Shear Interferometer for high angular resolution imaging. The program has been done jointly with Raman Research Institute (RRI), Bangalore.

A dual polarization receiver operating in the frequency range $85-115 \mathrm{GHz}$ has been installed on the 10.4 m millimeter wave telescope of RRI.

\subsection{Developments in observatories}

A few other progress reports received from observatory groups are summarized below:

The SOFIN Spectrograph has been constructed for the 2.5 Nordic Optical Telescope (La Palma, Canaries) to provide Spectroscopic observations with very high spectral resolution. The spectrograph has been designed and manufactured at the Crimean Astrophysical Observatory in collaboration with the observatory of Helsinki University. The Spectrograph has echelle grating, cross dispersion prism and three camera of 2000,1000 and $350 \mathrm{~mm}$ focal lengths. The maximum spectral resolution with the first camera is about $1,50,000$. It is installed at the Cassegrain focus at the telescope and operates under full remote control through the CAMAC interface from the Gateway-2000 personal computer. The Astromed CCD Cameras are used for spectra detection (Contributed by P.P.Petrov).

The TV complex using intensified isocon D Digital and $0.5 \mathrm{~m}$ telescope are in progress. TV photometry has been developed for objects upto $17^{\mathrm{m}}$ with exposure time 1-6 minutes and accuracy about $0^{m} .02$ digital TV Spectrophotometry optical unit is created by VI Pronik and L M Sharipova where astigmatism and coma have been suppressed in the slitless spectrograph using transparent gratings. The original digital TV Spectrograph of moderate dispersion created by A $\mathbf{N}$ Abramenko permits seeing spectra and slit images on the TV screen simultaneously. The TV method and apparatus for the photometrical observations of near-earth objects have been developed (Report by VV Prokof'eva)

\subsection{Optical fibres}

Use of optical fibres in astronomical instruments continued to increase. Remarkable advantages in manouvering beams in instruments like multiple object spectrographs are being fully utilised. Fibres in the improved transmission characteristics have been developed and many uses of these have been described in the previous section. Combination of images obtained by separate telescocpes are also being investigated in some novel projects. A special meeting on these topic was held during the period (3).

\subsection{Active and adaptive optics (adapted from a report by J.M.Beckers)}

The two functions of collection of large photon flux and concentrating them into sharp images by optical telescopes have undergone changes in their cycle of domination in optical astronomy. Initially the angular resolution was the dominant requirement, until the atmospheric seeing limit was reached, and then the collecting area and detection efficiency became the most important factor. With the introduction of techniques which overcame the limits set by atmospheric seeing the emphasis is changing back to angular 
resolution.

The $240 \mathrm{~cm}$ diameter Hubble Space Telescope has overcome the seeing limit by locating it above the earth's atmosphere. It is radical way of solving the atmospheric seeing problems. It is expensive and is almost certainly not affordable at least for some time to come for modern 8-10 meter diameter telescopes. Except for wavelengths inaccessible from ground astronomers and military programs have therefore, been developing techniques which makes diffraction limited imaging possible with earth based telescopes. One of these, speckle image construction has been very successful in giving diffraction limited images. The most powerful and promising technique for all astronomical applications is, however, the one using adaptive optics. For an unresolved image it puts most of the collected photons in as small an image as possible, which benefits in discriminating against the sky background both in high spectral and spatial resolution spectroscopy and in doing interferometric imaging. For resolved objects adaptive optics allows imaging without the complications of image reconstruction techniques applied to short exposure, noisy speckled images, thereby reaching to much fainter and more complex objects.

Modern large telescopes have been designed to give image qualities by themselves of a 0.2 arc seconds FWHM. A number of telescope imperfections contribute to this: like i) tracking errors ii) mechanical flexure of telescope structure supporting the optical components resulting in misalignment and aberrations of the optics iii) deviations from the perfect optical figure resulting from polishing errors, mirror support and thermal inhomogeneities in the mirror blanks, and iv) seeing generated by the telescope itself, by creating a temperature gradient in its vicinity.

Active optics is a technique which removes some of these. A good example is the ESO New Technology Telescope (NTT) where the shape of the rather thin primary mirror is controlled. A number of actuators in the mirror support adjusts its shape until the mirror figure, as measured by a Hartmann Shack wavefront sensor using a stellar image in the telescope field of view, is optimum. This correction is done with a time constant of a few minutes, so that only slow tracking errors, mechanical flexure effects, mirror support and large scale ( $>$ actuator spacing) optical figure errors are removed. This has resulted in a very good telescope image quality in which only fast tracking errors, small scale optical figure errors and mirror seeing remain to limit the telescope image quality. Active mirror supports are now being incorporated in all 8 to $10 \mathrm{~m}$ telescopes; it is an essential ingredient in the achievement of the 0.2 arc sec. FWHM image quality.

Adaptive optics is very similar to active optics in principle; the significant difference being that it works much faster, in the millisecond range. This removes not only the slowly changing telescope wavefront disturbances, but also the rapidly changing atmospheric variations.

Over the past few years a few astronomical systems using adaptive optics have become operational. These include the $3.6 \mathrm{~m}$ ESO telescope at $\mathrm{La}$ Silla, the Canada-France-Hawaii $3.6 \mathrm{~m}$ telescope at Mauna Kea, the 6.8 m MMT at Mount Hopkins, Arizona and a few other smaller systems. Full details of the activities are given in (4)

In adaptive optics, the necessity of using a resonably bright unresolved star becomes necessary. While some of them have utilized nearby stars in the field, a new idea of creating a small spot of light high up in the atmosphere has been successfully employed. The method is known as LGS (Laser Guide Star), where a strong laser beam is sent up after collimation by the telescope. The artificial stars are created by Rayleigh scattering of laser radiation off molecules and aerosols in the high (10-15 km) atmosphere. 
A still better idea for measuring the wave front distortions is to utilize the Mesospheric layer of neutral sodium atoms at around $90 \mathrm{~km}$ height, which can create a brighter and more distant light source by resonance scattering. Preliminary experiments with Na-LGS have been performed; their use in adaptive optics operations are expected shortly.

Even with the use of adaptive optics angular resolution achievable will be limited by telescope diameters. The largest telescopes with diameters of 8 to 10 meters, presently in existence or being planned approach probably the limit of what can be achieved in single mirror telescopes. The way out of this limitation is probably interferometric imaging with arrays of telescopes; major efforts are presently under way to implement interferometric imaging in optical astronomy. Interferometric arrays are in operation in Australia (Culgoora) England (Cambridge), France (CERGA) and in the USA (Mt. Wilson, MMT). Others are under construction or in advanced planning stages in Europe (the VLT interferometer or VLTI) and in the USA (Mt Hopkins, Anderson Mesa, the CHARA array, the Keck Interferometric array or KIA). Both the VLTI and the KIA involve the use of large 8-10 m telescopes to achieve the highest possible sensitivity; to perform optimally these telescopes will be equipped with adaptive optics.

Even with interferometric arrays angular resolution will be probably limited to milli-arc seconds because of atmospheric and possibly physical limitations. Interferometers in space or even on the moon have been proposed to remove these limitations. Indirect ways to achieve sub-milli arc seconds resolution can be found through temporal photometric spectroscopic and astrometric variations. It has been proposed to discuss some aspects of such experiments in the symposium No. S166 during XXII General Assembly in August 1994.

\section{Working groups}

Commission 9 has three working groups, Wide Field Imaging, Detectors and High Angular Resolution Interferometry. The name of the working group on Wide Field Imaging has been changed from its earlier one of Astronomical Photography, because of new developments in techniques. Reports about the activities of these groups are given below:

\subsection{Wide Field Imaging (Report by R.M.West, Chairman of the Working Group)}

The IAU Working Group on Wide Field Imaging (WG WFI) was established at the 1991 General Assembly in Buenos Aires. It is the successor of the WG on Astronomical Photography, but covers more areas Sky surveys and Patrols, Wide Field Detector (e.g. Photographic Techniques and CCD Mosaics), Digitization Techniques, Archival and Retrieval of Wide Field Data. By the end of June 1993 it had about 200 members and consultants. The organizing committee (OC) consists of: J. Guivert (Paris, France), R.M. Humphreys (Minneapolis, USA), K. Ishida (Tokyo, Japan), R. Kron (Chicago, USA), B.M. Lasker (Baltimore, USA) H. Lorenz (Potsdam, Germany), H.T. McGillivray (Edinburgh, UK, Secretary), D. Malin (Epping, Australia), N. Reid (Pasadena, USA), M. Tsvetkov (Sofia, Bulgaria) and R.M. West (Garching, Germany, Chairman). The WG WFI issues a semi-annual Newsletter (Editor, H.T. McGillivray).

Wide Field Imaging is characterized by the rapid and efficient acquisition of very large data sets. This leads to considerable handling problems, concerning both the initial extraction of relevant information for scientific interpretation, as well as the time- robust data storage and easy retrieval at a latter date. It is a major goal of the WG to bring together all available expertise on the related repidly 
progressing technology (e.g. panoramic detectors and storage media) and those major research projects which are dependent on large-scale observations (e.g. cataloguing and structural studies). The WG is particularly concerned with 1) integration of techniques and science, 2) coordination (e.g. of the photography and CCD groups), 3) standardization (procedures, formats etc.) and dissemination and sharing of information to the larger community.

The WG activities have included the organization of IAU Symposium 161 on "Astronomy from WideField Imaging" , (Potsdam, Germany, 23-27 August, 1993) with about 200 participants. A major WG Project (WFPA) is the registration in a computer readable form of the plate catalogues over the world (in-charge: M. Tsvetkov). A large fraction of plates in storage are now on file. The possibility of large scale digitisation of (a subset of) these plates is being studied.

A recent decision by Kodak to discontinue the production of several important astronomical emulsions, notably Ila and 103a, has led to severe problems for various current large scale projects. The WG now attempts to ensure the continued availability of at least the IIIa and IVN emulsions, and also to further development of a blue-sensitive Tech-Pan film similar to the $\mathbf{4 4 1 5}$ red-sensitive emulsion.

\subsection{Detectors (Report by M.Cullum, Chairman of the Working Group)}

The possibility of CCD detectors with 2048 x 2048 pixels was first mentioned in a Detector Working Group report in 1985 , although the available technology did not then permit the manufacture of useful devices of this size. In the intervening period CCD technology has steadily evolved and matured to the extent that today, chips of this format are becoming one of the mainstay optical detectors in all the major observatories.

Several trends can be observed in present developments, both on the part of manufacturers as well as users. These include the increased popularity of foundry runs, improvements in thinning techniques and back surface treatment, and the development of large mosaics.

Many of the large format CCDs currently in operation at the telescope have been produced by so- called silicon foundries. To date almost all of these have been produced by Loral, although other firms, such as Orbit, are now offering similar services. Instead of marketing individual tested CCD chips, foundries manufacture batches of wafers on a best effort basis according to standard or custom designs. Although it is likely that the majority of the chips produced in this way will not be suitable for astronomical applications, the significantly reduced unit cost and the generally acceptable production yields in recent years have made it worthwhile for many large institutes to go in this direction. A few have also designed their own CCDs to cater for special instrumental requirements. Even circular CCDs have been produced in this way.

The largest CCDs that are in widespread astronomical use have $2048 \times 2048$ pixels of 15 micron square. Larger single chips have been manufactured, and several institutes have tested chips of $4096 \times 4096$ pixels with 7.5 or 15 micron pixels. CCDs of the latter dimension are about the largest that can be accommodated within the 4 inch wafers used by many manufacturers. Efforts to achieve even larger formats have mainly concentrated on making mosaics of chips that are designed to be buttable with a gap of less than $0.5 \mathrm{~mm}$ between the butted sides. These devices may either be two-side buttable (either adjacent sides for $2 \times 2$ mosaics, or opposite sides for $1 \times n$ mosaics) or three sides (for $2 \times n$ mosaics). Several institutes are developing $2 \times 2$ mosaics of Loral chips giving $4096 \times 4096$ pixels (ESO, NOAO, 
IFA/Hawaii, RGO, amongst others). Existing plans even include a 2 x 5 mosaic with a total of 8190 x 10240 pixels for direct imaging applications (Japanese Nat. Obs.). For some specialised applications 'sparse mosaics' have been assembled using conventional CCD designs.

Major efforts have been made by a number of manufacturers and individuals to improve the sensitivity of CCDs, especially at UV wavelengths. Nevertheless, the fact that the large format CCDs now in use still have widely varying sensitivity figures show that these techniques have not yet been fully mastered. Of the major chip manufacturers only Tektronix produces thinned large format CCDs on a commercial basis, although even here the supplies have been erratic. EEV, Reticon and Thomson all have on-going thinning development programmes for their large format devices. M. Lesser (Steward Obs.) has successfully thinned Loral CCDs and supplied these to a number of observatories. It is worth noting here that if the rear surface has not received a permanent implant during manufacture, thinned CCDs usually require a UV flood prior to use to achieve a good short-wave sensitivity, thus adding to operational headaches. Also important for improving the sensitivity of a back-illuminated CCD is the anti-reflection coating. Both Tektronix and Lesser have demonstrated considerable advances in this area. Peak RQE figures exceeding $90 \%$ have been recorded in some cases, and over $50 \%$ at 300 $\mathrm{nm}$. Even $40 \% \mathrm{RQE}$ at $400 \mathrm{~nm}$ has been measured with front illuminated CCDs using the thin gate technology developed at JPL. Sadly, the RQE of CCDs in operational use at observatories today is generally significantly less than these figures suggest.

The readout noise of CCDs measured at the telescope at observatories around the world show a fairly consistent picture. Values in the range of $3-4 \mathrm{e}$ - are routinely observed with many recent types of CCD, with values up to 7 - 10 e- with some chip types. The use of mpp implants in many chip designs to reduce the dark current allows integrations of several minutes at room temperature.

There are several avenues of development that promise performance benefits in the near future. These include the improvement of skipper readout techniques to the point where single photo-electrons can be unambiguously detected (although at the expense of longer readout times), improved low signal CTE using super-notch designs (JPL), and advances in fast readout and/or low noise CCDs (EEV, JPL, Kodak, MIT Lincoln Labs, Sarnhoff). Fast parallel readout CCDs with smaller formats (32 x 32 to $256 \times 256$ ) are available from several manufacturers for applications such as fast guiding and wavefront sensing.

Developments in photon-counting detectors have generally been motivated by far UV applications or for those requiring high temporal resolution, for example adaptive optics and speckle interferometry. At the time of writing, the $1024 \times 1024$ MAMA detectors for the STIS have successfully passed the NASA technology demonstration test, and two engineering tubes are being assembled. The STIS will be equipped with two types of MAMA tube with solar blind CsI and Cs2Te cathodes. The DQE of the demonstration Cs2Te tube was $17 \%$ at $200 \mathrm{~nm}$. The MAMA is also available with visible light cathodes.

LEP in Paris has developed an electron bombarded CCD tube (EBCCD) which is able to distinguish up to 10 electrons per pixel per CCD readout, thus increasing the dynamic range by the same amount compared to a normal ICCD detector with the same frame rate. The device is presently employed as a wavefront sensor in the Come- On-Plus adaptive optics system at ESO. The format is $604 \mathrm{x}$ 288 pixels, although larger formats are planned. At the present time CCDs still cannot compete with photon-counting detectors for fast wavefront-sensing applications at very low signal levels, but the recent advances in fast readout CCDs indicate that this situation may change in the future. 
In the field of infrared array detectors substantial progress has been made during the last three years. Detectors with up to $256 \times 256$ pixels are now in fairly widespread use at observatories. In the wavelength range between 1 and 5 microns, two types of material are dominant: HgCdTe and InSb. Rockwell's $256 \times 256$ NICMOS-3 arrays (HgCdTe) are employed for wavelengths up to 2.5 microns. They have a pixel size of 40 microns, an RQE of $40-60 \%$, and a read noise of around 30 e- for a single readout, and down to $15 \mathrm{e}$ - with multiple non-destructive readouts. The full-well capacity is about $2.10^{5} \mathrm{e}-$. The dark current can be under $1 \mathrm{e}-/ \mathrm{sec} /$ pixel, but existing versions of these $256 \times 256$ devices have shown a slight persistence effect in which the dark current is dependent on the previous light exposure. This can be problematic, for example, in instruments that switch between imaging and spectroscopy on the same detector.

The SBRC $62 \times 58 \mathrm{InSb}$ array, which has been a workhorse for infrared astronomy over a number of years, has now been displaced by a new $256 \times 256$ array. This detector, which has 30 micron pixels, has a very high RQE of typically $80 \%$ out to 5 microns. The read noise, dark current and full well capacity are broadly similar to those of the NICMOS-3 detectors. Both the Rockwell and SBRC arrays are in use at many observatories, in many cases in instruments that have been specifically designed for the new large arrays.

Another InSb detector that has recently appeared on the market is a $256 \times 256$ array from Cincinnati Electronics. Although this device has not yet reached the astronomer, an interesting feature is the promised high well capacity of $10^{6} \mathrm{e}-$. Amber Engineering also produces an InSb array with $512 \times 512$ pixels, but this has a higher dark signal and is primarily intended for thermal imaging applications. Both Rockwell and SBRC have begun to develop 1024 x 1024 infrared arrays. One potential problem with arrays of this size is that the full-well capacity is likely to be similar to, or possibly even smaller than, present $256 \times 256$ arrays. With 16-times as many pixels to readout, this will require complex parallel readout electronics when used at longer wavebands.

In the mid-infrared region (10 - 30 microns), the situation has also changed rapidly. Doped silicon photoconductor arrays have now been displaced by more sensitive Impurity Band Conduction (or Blocked Impurity Band) detectors. Gallium, Arsenic or Antimony doped silicon devices are common, and the readout structures are similar to the near IR arrays. Aerojet Electrosystems has produced a 96 x 96 array, Hughes has made a 256 × 256 array, and Rockwell can provide 128 × 128 or $256 \times 256$ detectors. Available reports indicate that the performance of these detectors is excellent, although the larger devices have yet to be used on a telescope. Germanium IBC arrays are also being developed for the far infrared region.

Two major meetings on infrared array detectors took place in 1993 . The first of these was a SPIE meeting in Orlando, Florida in April on 'Infrared Detectors and Instrumentation'. The proceedings of this meeting are published as SPIE Vol. 1946 and edited by A Fowler. The second conference took place in July 1993 at UCLA under the title 'Infrared astronomy with arrays; the next generation'. The proceedings of this meeting will be edited by I. McLean and published by Kluwer by mid-1994. The IAU Symposium 167 ('Advances in Array Technology and Applications') will take place between $15-19$ August 1994, and will cover both optical and infrared array detectors. The Chairman of this symposium is D. Philip.

Much of the data on which this report is based was obtained from an e-mail survey carried out in September 1993. Thanks are due to the following who supplied information on the status of detectors 
at their respective institutes: J. Barton, A. Danks, G. Finger, J. Geary, F. Harris, O. Iwert, M. Iye, J. Janesick, P. Jordan, G. Lelievre, G. Luppino, I. McLean, R. Reed, P. Sinclaire, R. Stover and A. Walker Infrared detectors (Contributed by I.McLean)

The astronoshing progress in the development of infrared array detectors and their impact on astronomy was demonstrated in July 1993 at a conference entitled "Infrared Astronomy with Arrays": The News Generation organized by Prof Ian McLean and held at the University of California, Los Angeles (UCLA). Almost 300 registered participants from all over the world gathered to present observational results with arrays, describe new instruments and hear from detector manufacturers about the future prospects. About 180 papers were presented including invited reviews, contributed talks and posters. The conference covered astronomical applications of IR arrays, from near to the far infrared, new instruments, future detectors, space missions, the impact of IR astronomy on very large telescopes and even the importance of IR arrays for astronomy from Antarctica.

Infrared array detectors for wavelengths less than 5 microns now have $256 \times 256$ pixels, high QE ( $>$ $60 \%$ ) and low noise ( $<40$ electrons). Numerous cameras are in operation, such as the two color, twin detector system at UCLA. One of the highlights of the meeting was the extent to which high resolution $(\mathrm{R}>10,000)$ IR spectroscopy is benefitting from these sensitive devices. For longer wavelengths $(10-20)$ microns) where the problems of thermal background are worse, arrays of $128 \times 128$ pixels have arrived.

Manufacturers of near IR arrays (made from indium antimonide on from mercury-cadmium telluride) announced at the meeting that they plan to develop 1024 x 1024 pixels arrays within the next $2-3$ years. Noise performance is expected to reach 10 electrons or less.

In other words, infrared array detectors will provide a performance comparable with optical instruments, and many optical astronomy projects - especially spectroscopic ones - can now be carried out at infrared wavelengths. This is ideal for cooler sources, heavily reddened objects and galaxies at high redshifts.

The Proceedings of the conference at UCLA will be edited by McLean and published in 1994 by Kluwer.

\subsection{Working Group on High Angular Resolution Interferometry (Chairman, W.J.Tango)}

Major focus of activities in the WG has been organization of IAU symposium No. 158 on "Very High Angular Resolution Imaging" held on 11-15 January 1993 in Sydney, Australia. Principal topics discussed in the symposium cover imaging techniques, optical, radio and millimetre wave domains, speckle interferometry both in optical and infrared bands, and the results and future prospects of the experiments.

\section{Extra-visible band observations}

In the course of evolution of observational astronomy, many channels of information have been opened up, as new laboratory techniques of detecting and measuring these have been recognized and developed. This is not a new process; in the history of science we find opening up of IR channel (Herschel 1800), UV (Ritter 1801), Radio (Hertz 1888), X-rays (Roentzen 1896) and $\gamma$-rays (Rutherford 1901) in the electromagnetic spectra. As carriers of astronomical information even fundamental particle streams have come into the fold with cosmic rays (Hess 1912), solar wind (Bierman 1951) and neutrinoes (Davis 1966). The newly discovered channel of the gravitational waves (Weber 1962) has technically entered 
the area of astronomical techniques.

The recognition of the new channels by astronomers have been rather slow and hesitant. IR could not be effectively used until developments in the solid-state physics in the middle of the present century brought forth sensitive detectors; neither could the ultra-violet channel used before we could send the instruments and detectors beyond the absorbing layers of our atmosphere. Years after Jansky had stumbled upon the new sources through radio waves, the astronomers were slow to recognize the technique as a powerful one complementary to existing optical method.

Radio Astronomy was only a technique when started, but later astrophysics flooded the whole scene. The specialization which had distinguished the branch have largely disappeared. Many of the developments in the field e.g. image processing techniques have been adopted in other branches of astronomy; and several innovations in technique developed elsewhere have been assimilated in this branch. Logically the technique - part of Radio Astronomy should come under the fold of Instruments and Techniques, as it has happened in the case of IR astronomy. So also the innovations in High Energy Astrophysics techniques, which do not have a proper organized group in the IAU should logically be taken under the wings of Commission 9. A multinational collaborative project where development of a rocket-borne EUV imaging spectrograph for solar studies were needed now nearing completion (5); the first experiment is expected to be performed in late 1993 .

We have been approached by a group of particle physicists, to co-sponsor a meeting where astronomical techniques of detecting and measuring particle streams may be discussed. Such experiments have been performed by several astronomical groups and important bits of information about several forms of physical processes happening in the universe have been obtained. With the idea of the need to develop any new techniques of observation, which complements our existing methods, we have extended our co-sponsorship for the meeting. The meeting is going to be held in January 1994, in Bangalore, India.

\section{Acknowledgements}

In preparation of this report I have received help from many scientists, who had sent reports, reprints etc, based on which bulk of the report has been written; I am grateful to all of them. Wherever possible $I$ have acknowledged their help in the body of the report. I specially appreciate the useful advices sent by J. Davis, I. McLean, M. Cullum, R.M. West, C. Humphries, P. Mack, P. Felenbok, A. Bhatnagar and Shen Panan which have been of enormous help in my efforts. I am grateful to several of my colleagues for help and assistance in the preparation of this report; they include K.R. Sivaraman, S.K. Jain, A.K. Saxena, A.K. Pati, A. Vagiswari, K.T. Rajan, S.B. Ramesh and Pramila Mohan.

\section{References}

1. Perryman M.A.C., Foden C.L. and Peacock A: Nuclear Instruments and Methods in Physical Research, A 325 (1993)

2. Izv. Krim astrophys obs. $\underline{86},(1992)$

3. Optical Fibres in Astronomy II (Sydney, Australia, Nov 10-13, 1991)

4. J.M.Beckers, "Adaptive Optics for Astronomy: Principles, Performance and Applications" Ann. Rev. Astron \& Astrophys, 31, 1993.

5. S.K.Jain et al. High Resolution Extreme Ultraviolet (EUV) Spectrometer: XV meeting of Astronomical Society of India, Bombay (1993) 